\title{
Influenza, Like COVID-19, Needs Randomized Trials
}

\author{
Michael B. Rothberg, MD, MPH \\ Center for Value-Based Care Research, Cleveland Clinic Community Care, Cleveland Clinic, Cleveland, OH, USA.
}

J Gen Intern Med 36(6):1490-1

DOI: $10.1007 / \mathrm{s} 11606-020-06567-7$

(C) Society of General Internal Medicine 2021

$\mathrm{T}$ he COVID-19 pandemic has renewed interest in the treatment of viral respiratory infections. The severity of the infection and widespread nature of the illness have spurred unprecedented investment in vaccines and antivirals, although both remain elusive. In the face of a high mortality illness, with pressure to do something, it can be difficult to enroll patients in randomized trials, and doctors rely on observational studies, case reports, and personal experience. Once a treatment becomes accepted, or is even rumored to be effective, it can be difficult to study. Now, 10 months into the pandemic, with millions of patients treated, we have evidence that remdesivir, hydroxychloroquine, lopinavir/ritonavir, and interferon do not appear to meaningfully impact 28-day mortality. ${ }^{1}$ Studies are ongoing. Similarly, despite decades of research in influenza, randomized trials demonstrating reduced hospitalization or mortality are lacking.

The first antiviral medication for influenza, amantadine, was approved by the FDA in 1976. However, it was rarely used, because timely diagnosis of influenza was difficult and there were concerns about neuropsychiatric side effects. The advent of rapid diagnostic testing concurrent with the introduction of the neuraminidase inhibitors, oseltamivir and zanamivir in 1999, allowed for widespread diagnosis and treatment. Both drugs reduce the duration of symptoms by about $24 \mathrm{~h}$. This should not be minimized, because these hours come off the beginning of the illness, when patients feel the worst. However, because all studies were conducted in healthy volunteers, they have been underpowered to detect serious complications of influenza; thus, the impact on hospitalizations is unknown. Even CAPSTONE-2, which compared baloxavir, oseltamivir, and placebo for high-risk patients with uncomplicated influenza, was underpowered to detect differences in hospitalizations, with an overall hospitalization rate of only $1 \% .^{2}$

Perhaps more concerning, there are no randomized trials of patients hospitalized with influenza. In an eerie prelude to

Received October 21, 2020

Accepted December 22, 2020

Published online January 22, 2021
COVID-19, observational studies of oseltamivir during the H1N1 pandemic demonstrated improved outcomes, and randomized trials were never conducted. More than a decade later, the CDC still recommends antiviral treatment for all hospitalized patients as well as those at high risk of complications, and it would probably be considered unethical to perform a placebo-controlled trial. Moreover, the mortality rate for patients hospitalized with influenza is low, so any study with mortality as an endpoint would have to be large. Fortunately, results of a randomized trial of baloxavir for hospitalized patients, all of whom also received oseltamivir, should be available soon. ${ }^{3}$

Despite the CDC recommendations, most patients with influenza are not treated. Two significant barriers are cost and timely diagnosis. A 5-day course of Tamiflu costs about $\$ 180$, and even generic oseltamivir costs at least $\$ 40$ (GoodRx cash price). The only other oral therapy, single-dose baloxavir, costs $\$ 156$. Generic amantadine, while inexpensive, is not currently recommended due to viral resistance. An additional generic treatment would be welcome, and elderberry extract, which is readily available and inexpensive, is a promising candidate. Elderberry (Sambucas nigra) has been used in medicine for centuries to treat conditions ranging from respiratory infections to cardiovascular disease to constipation. ${ }^{4}$ It has in vitro activity against influenza A and B and several small randomized trials suggest that it could be effective in treating influenza illness, shortening symptoms by up to 4 days. ${ }^{5}$ In a recent article in JGIM, Macknin et al. ${ }^{6}$ reported on a randomized trial of elderberry extract for children and adults with influenza presenting to an emergency room.

The study included 87 patients, making it the largest study of elderberry extract to date. The authors are to be commended on the rigor of the study design. All patients had influenza diagnosed by PCR and at least 2 moderately severe influenza symptoms which had been present for $<48 \mathrm{~h}$. This point is important because the first 2 days of infection are characterized by rapid viral replication and antiviral therapies initiated outside that window are generally not effective. Although the authors intended to enroll only high-risk patients, after 1 month, they expanded enrollment to include all patients with influenza, highlighting the challenge of studying high-risk patients.

The study was double-blinded, with patients receiving $15 \mathrm{ml}$ of liquid Sambucol ${ }^{\circledR}$ or an identical placebo for 5 days, 2-4 times daily depending on age. The majority of patients in both groups believed they were receiving the active therapy. 
The dosage and manufacturer were chosen for comparability to past elderberry trials, while the study design was meant to emulate the oseltamivir trials. Patients took more than $90 \%$ of the prescribed doses, and less than 5\% were lost to follow-up. Perhaps most important, the study was conducted entirely with philanthropic funds; the manufacturer was not involved.

As often occurs in well-designed trials of promising therapies, the treatment produced no benefit. Average duration of symptoms was identical in both groups. Interestingly, about half the patients were also treated with oseltamivir, which was associated with the expected 1-day reduction in symptoms. When stratified by receipt of oseltamivir, those not taking oseltamivir actually had longer duration of symptoms when treated with elderberry vs. placebo. Because the study was powered to detect a 2-day reduction in symptom duration, based on previous trials, it is possible that a smaller benefit could have been missed due to type II error. The more likely explanation is that elderberry is ineffective as a treatment for influenza, leaving oseltamivir and baloxavir as the only effective oral therapies.

The other obstacle to treatment is timely diagnosis. For all patients, the CDC suggests a strategy of treat first, diagnose later, as treatment after even $24 \mathrm{~h}$ is markedly less effective. If administered within $24 \mathrm{~h}$, both oseltamivir and baloxavir can shorten the course of illness by $33 \mathrm{~h}$ vs. $13 \mathrm{~h}$ for those who receive it later. ${ }^{7}$ Prior to COVID-19, during a local epidemic, influenza could be reliably diagnosed based on symptoms alone. In that setting, patients with fever and cough have a $70 \%$ chance of having influenza. ${ }^{8}$ This year, and perhaps for several years to come, rapid testing will be required to differentiate influenza from COVID-19.

The COVID-19 pandemic has taught us important lessons about caring for patients with viral respiratory illness. There has been massive expansion of virtual care, allowing for faster, safer diagnosis. Patients no longer have to make a doctor's appointment, find an open urgent care, or pay an emergency room fee to receive a timely diagnosis. Telemedicine provides instant medical access from anywhere, at any time. ${ }^{9}$ Moreover, keeping infectious patients out of crowded waiting rooms means less chance of spreading the virus, including COVID-19. This coming flu season, we can take advantage of the drive-through infrastructure developed for COVID-19 testing in order to deploy rapid tests for both diseases.

The more important lesson, however, is that we need placebo-controlled randomized trials, particularly in the face of a pandemic illness. Thanks to Dr. Macknin and colleagues, we can confidently advise patients not to take elderberry. We need similarly rigorous studies to understand what works for patients who are at high risk of complications and those hospitalized with life-threatening illness.

Corresponding Author: Michael B. Rothberg, MD, MPH; Center for Value-Based Care Research, Cleveland Clinic Community Care, Cleveland Clinic, Cleveland, OH, USA (e-mail: Rothbem@ccf.org).

\section{Compliance with Ethical Standards:}

Conflict of Interest: The author declares that he does not have a conflict of interest.

\section{REFERENCES}

1. Pan H, Peto R, Karim QA, et al. Repurposed antiviral drugs for COVID-19 -interim WHO SOLIDARITY trial results. MedRxiv 2020.10.15.20209817 [Preprint]. 2020 [cited 2020 Nov 18]. Available from: https://www.medrxiv. org/content/10.1101/2020.10.15.20209817v1 doi: https://doi.org/10. $1101 / 2020.10 .15 .20209817$

2. Ison MG, Portsmouth S, Yoshida Y, et al. Early treatment with baloxavir marboxil in high-risk adolescent and adult outpatients with uncomplicated influenza (CAPSTONE-2): a randomised, placebo-controlled, phase 3 trial. Lancet Infect Dis. 2020;20(10):1204-14. Clinicaltrials.gov registration number: NCT02949011.

3. Clinicaltrials.gov [Internet]. Study to Assess Efficacy and Safety of Baloxavir Marboxil In Combination With Standard-of-Care Neuraminidase Inhibitor In Hospitalized Participants With Severe Influenza. Clinical Trial; [cited 2020 Nov 18]. Available from: https://clinicaltrials.gov/ct2/show/ NCT03684044 Clinicaltrials.gov Identifier: NCT03684044.

4. Ulbricht $\mathbf{C}$, Basch $\mathbf{E}$, Cheung $\mathbf{L}$, et al. An evidence-based systematic review of elderberry and elderflower (Sambucus nigra) by the Natural Standard Research Collaboration. Journal of dietary supplements. 2014;11(1):80-120.

5. Zakay-Rones Z, Thom E, Wollan T, Wadstein J. Randomized study of the efficacy and safety of oral elderberry extract in the treatment of influenza A and B virus infections. J Int Med Res. 2004;32(2):132-40.

6. Macknin M, Wolski K, Negrey J, Mace S. Elderberry Extract Outpatient Influenza Treatment for Emergency Room Patients Ages 5 and Above: a Randomized, Double-Blind, Placebo-Controlled Trial. J Gen Intern Med. 2020;35(11):3271-7. Clinicaltrials.gov registration number: NCT03410862.

7. Hayden FG, Sugaya N, Hirotsu N, et al. Baloxavir Marboxil for Uncomplicated Influenza in Adults and Adolescents. N Engl J Med. 2018;379(10):913-23. Clinicaltrials.gov resgistration number: NCT02954354.

8. Zambon M, Hays J, Webster A, Newman R, Keene O. Diagnosis of influenza in the community: relationship of clinical diagnosis to confirmed virological, serologic, or molecular detection of influenza. Arch Intern Med. $2001 ; 161(17): 2116-22$.

9. Rothberg MB, Martinez KA. Influenza Management via Direct to Consumer Telemedicine: an Observational Study. J Gen Intern Med. 2020;35(10):3111-3.

Publisher's Note: Springer Nature remains neutral with regard to jurisdictional claims in published maps and institutional affiliations. 